\title{
MDMA impairs mitochondrial neuronal trafficking in a Tau- and Mitofusin2/Drp1-dependent manner
}

\author{
Daniel José Barbosa $\cdot$ Román Serrat • Serena Mirra • Martí Quevedo • \\ Elena Gómez de Barreda $\cdot$ Jesús Ávila $\cdot$ Eduarda Fernandes · \\ Maria de Lourdes Bastos $\cdot$ João Paulo Capela $\cdot$ Félix Carvalho $\cdot$ Eduardo Soriano
}

Received: 12 November 2013 / Accepted: 29 January 2014 / Published online: 13 February 2014

(C) Springer-Verlag Berlin Heidelberg 2014

\begin{abstract}
Identification of the mechanisms by which drugs of abuse cause neuronal dysfunction is essential for understanding the biological bases of their acute and longlasting effects in the brain. Here, we performed real-time functional experiments of axonal transport of mitochondria to explore the role of in situ mitochondrial dysfunction in 3,4-methylenedioxymethamphetamine (MDMA; "ecstasy")-related brain actions. We showed that MDMA dramatically reduced mitochondrial trafficking in hippocampal neurons in a Tau-dependent manner, in which glycogen synthase kinase $3 \beta$ activity was implicated. Furthermore, we found that these trafficking abnormalities were rescued by over-expression of Mitofusin2 and dynamin-related protein 1, but not of Miro1. Given the relevance of mitochondrial targeting for neuronal function and
\end{abstract}

Daniel José Barbosa and Romàn Serrat have contributed equally to this work.

Félix Carvalho and Eduardo Soriano are co-senior authors.

Electronic supplementary material The online version of this article (doi:10.1007/s00204-014-1209-7) contains supplementary material, which is available to authorized users.

D. J. Barbosa · M. L. Bastos · J. P. Capela ·

F. Carvalho $(\bowtie)$

REQUIMTE (Rede de Química e Tecnologia),

Toxicology Laboratory, Department of Biological Sciences,

Faculty of Pharmacy, University of Porto, Rua Jorge Viterbo

Ferreira 228, 4050-313 Porto, Portugal

e-mail: felixdc@ff.up.pt

D. J. Barbosa $\cdot$ R. Serrat $\cdot$ S. Mirra $\cdot$ M. Quevedo .

E. Soriano $(\square)$

Developmental Neurobiology and Regeneration Lab,

Institute for Research in Biomedicine, Barcelona Science Park,

C/Baldiri I Reixac 10-12, 08028 Barcelona, Spain

e-mail: esoriano@ub.edu neurotransmission, our data underscore a novel mechanism of action of MDMA that may contribute to our understanding of how this drug of abuse alters neuronal functioning.

Keywords 3,4-Methylenedioxymethamphetamine (MDMA; "ecstasy") · Mitochondrial trafficking · Mitochondrial dysfunction · Drugs of abuse · Neurotoxicity

\section{Introduction}

Drugs of abuse cause a variety of complex cellular events, including changes in membrane excitability and neurotransmission, activation of complex signalling pathways, altered synaptic physiology and structural changes, and drug-evoked synaptic plasticity and neurotoxicity, which mediate both acute and long-term effects and addiction (Capela et al. 2009; Kenny 2011; Lüscher and Malenka 2011). Within this context, 3,4-methylenedioxymethamphetamine (MDMA; "ecstasy") is a major drug of abuse with neurotoxic properties well documented in both humans and laboratory animals (Bai et al. 2001; Capela

\footnotetext{
D. J. Barbosa $\cdot$ R. Serrat $\cdot$ S. Mirra $\cdot$ M. Quevedo $\cdot$ E. Soriano Department of Cell Biology, University of Barcelona, Barcelona, Spain

D. J. Barbosa $\cdot$ R. Serrat · S. Mirra $\cdot$ M. Quevedo

E. Gómez de Barreda · J. Ávila · E. Soriano

Centro de Investigación Biomédica en Red sobre Enfermedades

Neurodegenerativas (CIBERNED, ISCIII), Madrid, Spain

E. Gómez de Barreda · J. Ávila

Center of Molecular Biology Severo Ochoa (CSIC-UAM),

Campus de la Universidad Autónoma de Madrid, C/Nicolás

Cabrera 1, 28049 Madrid, Spain
} 
et al. 2009; Granado et al. 2011), as well as in several in vitro neuronal models (Barbosa et al. 2012, 2014a, 2014b; Capela et al. 2006, 2007, 2013), in which mitochondrialdependent pathways seem to play a key role (Alves et al. 2007, 2009b; Puerta et al. 2010). Nevertheless, the mechanisms underlying drug's neurotoxicity remain incompletely understood.

In neurons, the high dependence on mitochondria for local adenosine 5'-triphosphate (ATP) supply and $\mathrm{Ca}^{2+}$ buffering in neurotransmission requires highly regulated mitochondrial trafficking and targeting (López-Doménech et al. 2012; Sheng and Cai 2012). Axonal transport of mitochondria is mediated by motor proteins: members of the kinesin family mediate anterograde transport, whereas cytoplasmic dyneins mediate retrograde transport, processes highly dependent on ATP produced locally by mitochondria (MacAskill and Kittler 2010; Shahpasand et al. 2012; Sheng and Cai 2012). Kinesin-1, the principal plus-end-directed motor, is linked to the mitochondrial outer membrane proteins Miro1/2 by the adaptor protein Milton/Trak2 (Macaskill et al. 2009; Wang and Schwarz 2009). Miro $1 / 2$, guanosine 5 'triphosphatase (GTPase) proteins with two $\mathrm{Ca}^{2+}$-binding domains ( $\mathrm{EF}$ hands), regulate the integrity of this complex, by a $\mathrm{Ca}^{2+}$-dependent mechanism (Byun et al. 2012; Macaskill et al. 2009; Wang and Schwarz 2009).

Microtubule-associated protein Tau, which is primarily localized within the axonal compartment of neurons (Rintoul and Reynolds 2010), binds to microtubules, resulting in the formation of microtubule bundles. In neurons, Tau phosphorylation has been proposed to decrease mitochondrial transport (Rintoul and Reynolds 2010; Vossel et al. 2010). MDMA has been associated with ATP depletion (Capela et al. 2007) and increased Tau phosphorylation (Busceti et al. 2008), which suggests a possible interference of MDMA on mitochondrial trafficking.

Disruption of mitochondrial trafficking or of the dynamic equilibrium between mitochondrial fusion and fission may contribute to important neuronal deficits and herald neuronal injury or death (Perier and Vila 2012; Wang et al. 2013). Furthermore, mitochondrial dysfunction, including trafficking, has been linked to several psychiatric diseases (Atkin et al. 2013; MacAskill et al. 2010; Shao et al. 2008). In the present study, we evaluated whether mitochondrial dynamics, namely mitochondrial neuronal

\footnotetext{
E. Fernandes

REQUIMTE, Laboratory of Applied Chemistry, Department of Chemical Sciences, Faculty of Pharmacy, University of Porto, Rua Jorge Viterbo Ferreira 228, 4050-313 Porto, Portugal

J. P. Capela

Faculty of Health Sciences, University Fernando Pessoa, Praça 9 de Abril 349, 4249-004 Porto, Portugal
}

trafficking, is affected by MDMA. We showed that acute MDMA incubation substantially altered mitochondrial trafficking in hippocampal neurons. These findings underscore a novel mechanism of action of MDMA that may have important consequences in neuronal function.

\section{Materials and methods}

\section{Materials}

All reagents used were of analytical grade or of the highest grade available. Neurobasal medium, B27 supplement, penicillin/streptomycin $10,000 \mathrm{U} / \mathrm{mL}$, GlutaMAX ${ }^{\mathrm{TM}}$ supplement, $0.5 \%$ trypsin $/ 1 \mathrm{mM}$ ethylenediaminetetraacetic acid (EDTA) without phenol red, horse serum, Opti-MEM medium, Lipofectamine 2000, Dulbecco's phosphatebuffered saline (DPBS) and 3-(4,5-dimethyl-2-thiazolyl)2,5-diphenyl-2H-tetrazolium (MTT) bromide were purchased from Gibco Laboratories (Lenexa, KS, USA). Poly-L-lysine (PLL), triton $\times-100, \mathrm{SB} 415286, \mathrm{Na}_{3} \mathrm{VO}_{4}$, $\mathrm{NaF}$, Tris- $\mathrm{HCl}$, bromophenol blue and $\beta$-mercaptoethanol were obtained from Sigma-Aldrich (St. Louis, MO, USA). DNase recombinant $I$ and complete protease inhibitor cocktail were from Roche Diagnostics (Mannheim, Germany). Fluo4/AM was purchased from Molecular Probes (Invitrogen Inc, Carlsbad, CA, USA). Mouse anti- $\alpha$-actin monoclonal antibody was supplied by Calbiochem (Darmstadt, Germany). Swine anti-rabbit and goat anti-mouse IgG-peroxidase polyclonal antibodies were purchased from DAKO (Glostrup, Denmark). MDMA (hydrochloride salt) was extracted and purified from high-purity MDMA tablets kindly provided by the Portuguese Police Department. The salt obtained was pure and fully characterized by NMR and mass spectrometry methodologies.

Animals, cell culture and transfection

All procedures were approved by the Barcelona Science Park's Animal Experimentation Committee and performed in compliance with the "Guide for the Care and Use of Laboratory Animals" of the Institute for Laboratory Animal Research (Institute for Laboratory Animal Research 2011), and the European Community Council's directive and the National Institute of Health's guidelines for the care and use of laboratory animals. Wt OF1 embryos and C57BL/6 Tau (-/-) embryos (de Barreda et al. 2010), at embryonic day (E) 15-E16 (the mating day was E0), were used. Primary hippocampal cultures were prepared following previously published methods (Llorens-Martín et al. 2011; López-Doménech et al. 2012). Briefly, embryos were placed in ice-cold DPBS (-/-) containing $0.6 \%$ glucose, and the hippocampi were dissected out. Following trypsinization 
(0.05\% trypsin/EDTA) and DNase I treatment, tissue pieces were dissociated, and cells were seeded at a density of 78,000 cells $/ \mathrm{cm}^{2}$ in $35-\mathrm{mm}$ Fluorodish plates (World Precision Instruments, Sarasota, FL, USA) precoated, overnight, with $0.2 \mathrm{mg} / \mathrm{mL}$ PLL solution (prepared in $0.1 \mathrm{M}$ borate buffer, $\mathrm{pH} 8.5$ ), for studies of mitochondrial trafficking. For Western blotting, cells were seeded onto 6-well precoated (0.05 mg/mL PLL solution, overnight) plates. To evaluate mitochondrial length, hippocampal cultures were seeded in $12-\mathrm{mm}$ round precoated $(0.2 \mathrm{mg} / \mathrm{mL}$ PLL solution, overnight) glass coverslips. Cultures were maintained at $37{ }^{\circ} \mathrm{C}$ in a $5 \% \mathrm{CO}_{2} / 95 \%$ air atmosphere. Transfection was performed at 4 days in vitro (DIV) using Lipofectamine 2000, and cultures were used at 6DIV. Cultures were transfected with MitDsRed (a gift from Antonio Zorzano) and GFP or co-transfected with one of the following constructs: Miro1, Miro1 $\Delta \mathrm{EF}$ (Macaskill et al. 2009), GSK3 $\beta$ Aln9 (Byun et al. 2012), Mitofusin2, Mitofusin2 R94Q (Misko et al. 2010) or dynamin-related protein 1 (Drp1) K38A (Zhao et al. 2011). The lack of Tau protein in hippocampal cultures prepared from C57BL/6 Tau (-/-) embryos was confirmed by Western blotting (data not shown).

\section{Design of the study}

Hippocampal cultures were initially exposed to MDMA (1.6 $\mathrm{mM}$ ) for 45-120 min. The MDMA concentration used in this study is in accordance with other studies performed during short incubation periods (Capela et al. 2006, 2013; Carvalho et al. 2004; Simantov and Tauber 1997). Ricaurte et al. (2000), using an adjustment for body mass/ surface area and drug's clearance, calculated the equivalent dose in humans of a $20 \mathrm{mg} / \mathrm{kg}$ rat dose (an accepted neurotoxic dose to this species) to be $1.28 \mathrm{mg} / \mathrm{kg}$ or approximately $96 \mathrm{mg}$ in a $75 \mathrm{~kg}$ individual. Human MDMA users typically use MDMA single doses of 75-125 mg. The fact that some individuals report using up to 10-25 individual tablets of MDMA per occasion (Parrott 2005) further suggests that there is little or no margin of safety between the recreationally used and neurotoxic doses of MDMA. In our experimental conditions, MDMA (1.6 mM) did not induce significant toxicity, as evaluated by the MTT reduction assay (data not shown). Exposure to MDMA (1.6 mM) for $90 \mathrm{~min}$ was selected for all the remaining studies of mitochondrial transport. Tau phosphorylation levels and mitochondrial length were also evaluated after exposure to MDMA (1.6 mM) for $90 \mathrm{~min}$.

Live-imaging of axonal transport of mitochondria and intracellular $\mathrm{Ca}^{2+}$ levels in hippocampal neurons

Live-imaging of hippocampal neurons was performed using a Olympus IX81 confocal microscope (Olympus
Corporation, Tokyo, Japan), equipped with a $60 \times$ immersion objective, and a Andor Revolution ${ }^{\circledR}$ XD spinning disk confocal microscopy system composed of a iXonEM + EM-CCD camera (Andor Technology, Belfast, United Kingdom) and FRAPPA and solid-state laser combiner (ALC-UVP 350i, Andor Technology) with the renowned CSU-X1 confocal scanner (Yokogawa Electric Corporation, Tokyo, Japan). All cultures were kept at $37{ }^{\circ} \mathrm{C}$, using a heating insert on the microscope stage and an incubating chamber. For measurements of axonal transport of mitochondria, axonal processes were identified following morphological criteria. Time-lapse series of image stacks composed of 151 images $(512 \times 512$ pixels $)$ were taken every $6 \mathrm{~s}$, for $15 \mathrm{~min}$. All 151 images obtained were processed mainly with Andor IQ2 software (Andor Technology). Further image processing, analysis and video compilation and edition were done with Fiji (Image JA v1.45b, Open source software, NIH, Bethesda, Maryland, USA). Kymographs were generated with MetaMorph Software (Molecular Devices-MDS Analytical Technologies, Sunnyvale, CA, USA). Velocities of moving mitochondria were measured from the corresponding kymographs. In all cases, mitochondria were considered motile when they moved more than $5 \mu \mathrm{m}$ during the 15 -min periods. When a single mitochondrion moved in both the anterograde and retrograde directions, it was included in both groups.

For the measurements of intracellular $\mathrm{Ca}^{2+}$ levels, primary hippocampal cultures were loaded with Fluo4/ AM. These cultures were washed once with Krebs buffer (145 mM NaCl, $5 \mathrm{mM} \mathrm{KCl,} 2.5 \mathrm{mM} \mathrm{CaCl} 2,1 \mathrm{mM} \mathrm{MgCl}$, $5.6 \mathrm{mM}$ glucose, $10 \mathrm{mM}$ Hepes, $\mathrm{pH}$ 7.4) and subsequently loaded with $5 \mu \mathrm{M}$ Fluo4/AM, in Krebs buffer containing $0.4 \%$ pluronic acid (w/v), for $30 \mathrm{~min}$, at $37{ }^{\circ} \mathrm{C}$. Stock solution of Fluo4/AM $(250 \mu \mathrm{M})$ was prepared in DMSO. After this incubation time, the cultures were incubated in fresh Krebs buffer for a further $5 \mathrm{~min}$ (to allow the Fluo4/AM deesterification) and subsequently imaged. Time-lapse series of image stacks composed of 101 images $(512 \times 512$ pixels) were taken every $1 \mathrm{~min}$, during $100 \mathrm{~min}$. After $10 \mathrm{~min}$ of imaging, MDMA was added to the cultures, and images were taken again for another 90 min (images were continuously acquired before and after drug exposure).

Measurements of mitochondrial length

Hippocampal cultures transfected with MitDsRed and GFP and incubated with MDMA for 90 min were then fixed in $4 \%$ paraformaldehyde for $20 \mathrm{~min}$ at room temperature. Stacks of images $(1,024 \times 1,024$ pixels $)$ were acquired in a Leica TCS SP2 confocal microscope (Leica Microsystems, Mannheim, Germany), equipped with a $60 \times$ immersion objective and a Leica Confocal Software (Leica Microsystems). Further image processing and analysis 
was done with Fiji (NIH) by determining mitochondrialtagged MitDsRed. Mitochondrial length was estimated for each mitochondrion. To identify axonal processes, GFP was excited at $488 \mathrm{~nm}$ and mitochondria were visualized at $561 \mathrm{~nm}$.

Western blotting analysis of Tau phosphorylation

Cellular proteins were extracted with lysis buffer $[50 \mathrm{mM}$ Tris-HCl pH 7.5, $150 \mathrm{mM} \mathrm{NaCl}, 5 \mathrm{mM}$ EDTA, $1 \%$ triton $\times-100(\mathrm{v} / \mathrm{v}), 10 \%$ glycerol (v/v), complete protease inhibitor cocktail, $1 \mathrm{mM} \mathrm{Na} \mathrm{VO}_{4}, 10 \mathrm{mM} \mathrm{Na} \mathrm{P}_{2} \mathrm{O}_{7}$ and $10 \mathrm{mM}$ $\mathrm{NaF}$ ), followed by an incubation on ice for $30 \mathrm{~min}$. After centrifugation at $16,000 \mathrm{~g}$ for $10 \mathrm{~min}$ at $4{ }^{\circ} \mathrm{C}$, an SDSPAGE reducing buffer $[150 \mathrm{mM}$ Tris- $\mathrm{HCl} \mathrm{pH} 6.8,3 \%$ SDS (w/v), $30 \%$ glycerol (v/v), $0.075 \%$ bromophenol blue (w/v) and $15 \% \beta$-mercaptoethanol (v/v)] was added to the samples (1:2 ratio), which were then boiled at $90{ }^{\circ} \mathrm{C}$ for 5 min. Proteins were loaded and separated in $10 \%$ SDS/ polyacrylamide gels, at a constant voltage of $120 \mathrm{mV}$. Gels were transferred to nitrocellulose membranes (Whatman, Dassel, Germany) using a transfer buffer (20\% methanol $(\mathrm{v} / \mathrm{v})$ in $20 \mathrm{mM}$ Tris base and $139 \mathrm{mM}$ glycine) at $125 \mathrm{mV}$ for $1.5 \mathrm{~h}$. Membranes were then rinsed in Tris-buffered saline solution with Tween 20 [TBS-T $10 \mathrm{mM}$ Tris base, $140 \mathrm{mM} \mathrm{NaCl}$ and $0.1 \%$ Tween $20(\mathrm{v} / \mathrm{v}), \mathrm{pH} 7.4]$, and nonspecific sites were blocked for $1 \mathrm{~h}$ at room temperature in blocking buffer [3 \% BSA (w/v) in TBS-T]. Membranes were incubated with primary antibodies (overnight at $\left.4{ }^{\circ} \mathrm{C}\right)$ : rabbit polyclonal anti-P-Tau Thr181 $(1: 5,000)$ or mouse monoclonal anti- $\alpha$-actin $(1: 100,000)$. After washing five times (10 min each) with TBS-T, membranes were then incubated with secondary antibodies $(1 \mathrm{~h}$ at room temperature): swine anti-rabbit or goat anti-mouse IgG-peroxidase antibodies $(1: 2,500)$. All antibodies were diluted in blocking buffer. Following three washes (10 min each) with TBS-T, bands were visualized using ECL chemiluminescence reagents (Amersham Pharmacia Biotech, Buckinghamshire, UK), according to the supplier's instructions, and were quantified using the Quantity One software (Bio-Rad Laboratories, Hercules, CA, USA).

Data analysis

Values are represented as mean \pm SEM. Seven to sixteen axons, from 3 to 7 independent experiments, were analyzed for each condition. Normality of the data distribution was assessed by three tests: KS normality test, D'Agostino and Pearson omnibus normality test and Shapiro-Wilk normality test. In experiments with only one variable, statistical comparisons between groups were performed with MannWhitney test or unpaired Student's $t$ test (to compare two groups), or with Friedman test (nonparametric one-way ANOVA with repeated measures) to compare three or more
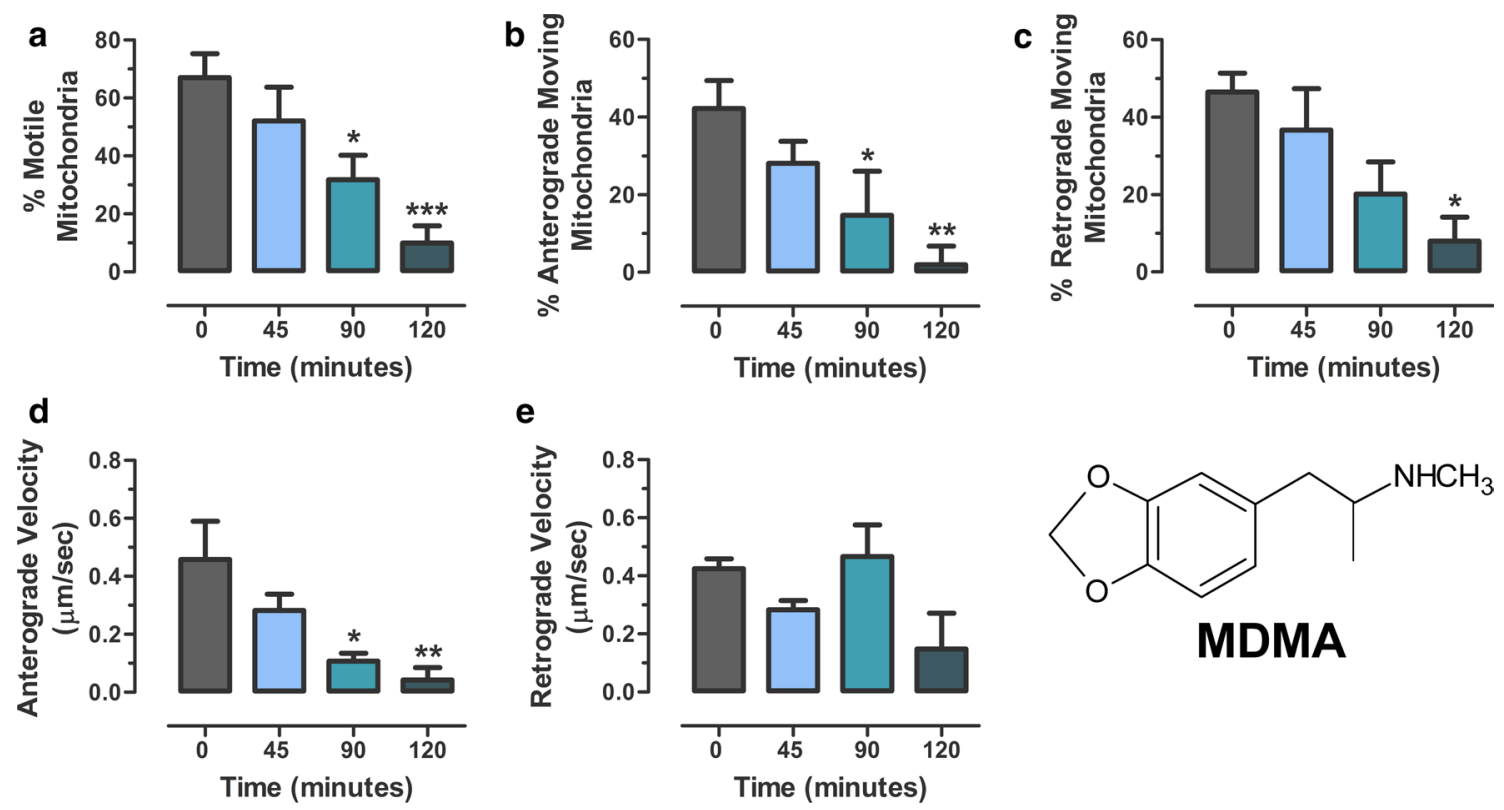

Fig. 1 Effects of MDMA on axonal transport of mitochondria in hippocampal neurons. Hippocampal neuronal cultures were transfected with MitDsRed and GFP at 4DIV, and axonal transport of mitochondria was measured at 6DIV. After imaging under control conditions, MDMA (1.6 mM) was added to the cultures, and axons were imaged again after 45, 90 and $120 \mathrm{~min}$ of incubation. a-e Graphical repre-

sentation of the percentage of motile mitochondria and velocities (both anterograde and retrograde directions) measured in kymographs obtained under control conditions and after exposure to MDMA $(1.6 \mathrm{mM})$. Data represent mean \pm SEM $(* p<0.05 ; * * p<0.01$; $* * * p<0.001$, MDMA versus control, Friedman test and Dunn's post hoc test) 
groups. Dunn's test was used for post hoc evaluations. To compare data from experiments with two variables, twoway ANOVA was used, followed by Bonferroni's test for post hoc evaluations. Details of the statistical analysis performed are described in each figure legend. All analyses were performed using GraphPad Prism 6.0 for Windows (GraphPad Software, San Diego, CA, USA).

\section{Results}

To address whether MDMA alters mitochondrial dynamics, we transfected hippocampal neurons with MitDsRed, performed video recordings of mitochondrial trafficking a
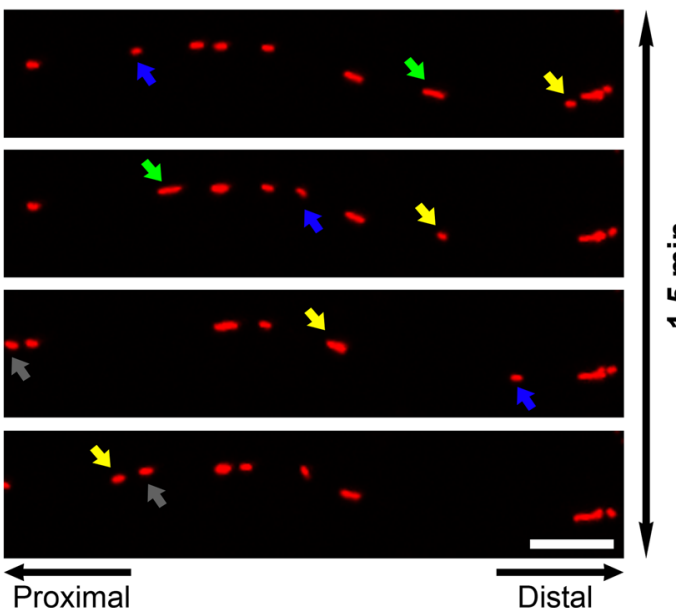

C

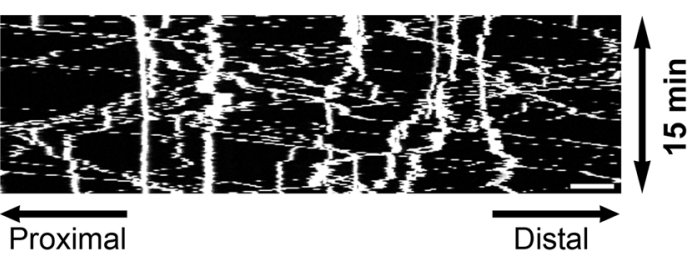

and generated kymographs, which were analyzed as described elsewhere (López-Doménech et al. 2012). Control neurons exhibited prominent mitochondrial trafficking (Figs. 1, 2), with an average of $70 \%$ of motile mitochondria, moving in both anterograde and retrograde directions at a speed of about $0.4 \mu \mathrm{m} / \mathrm{s}$ (Figs. 1, 2 and Supplementary Movie 1). Exposure to MDMA (1.6 mM) for 45,90 or 120 min dramatically reduced the mitochondrial trafficking in a time-dependent manner (Fig. 1). According to these results, the 90-min time-point was selected for all the remaining studies of mitochondrial transport. After 90 min of exposure to MDMA (1.6 mM), a dramatic reduction in the percentage of motile mitochondria (in both anterograde and retrograde directions), as well as in

b
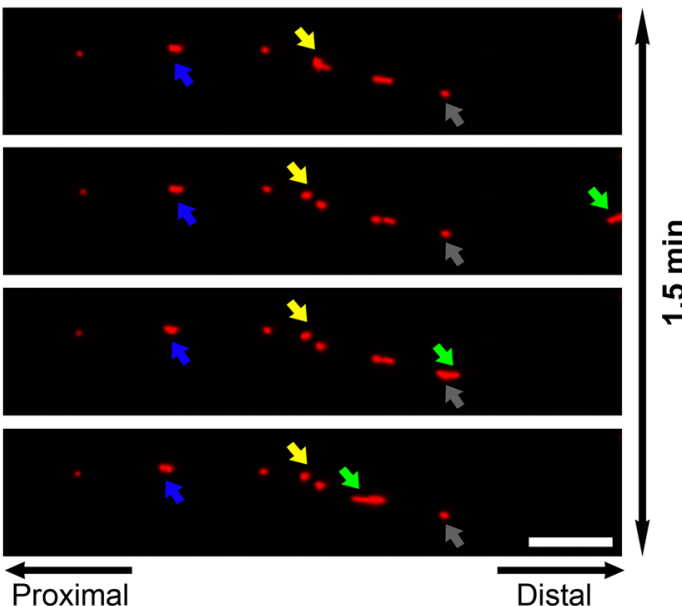

d
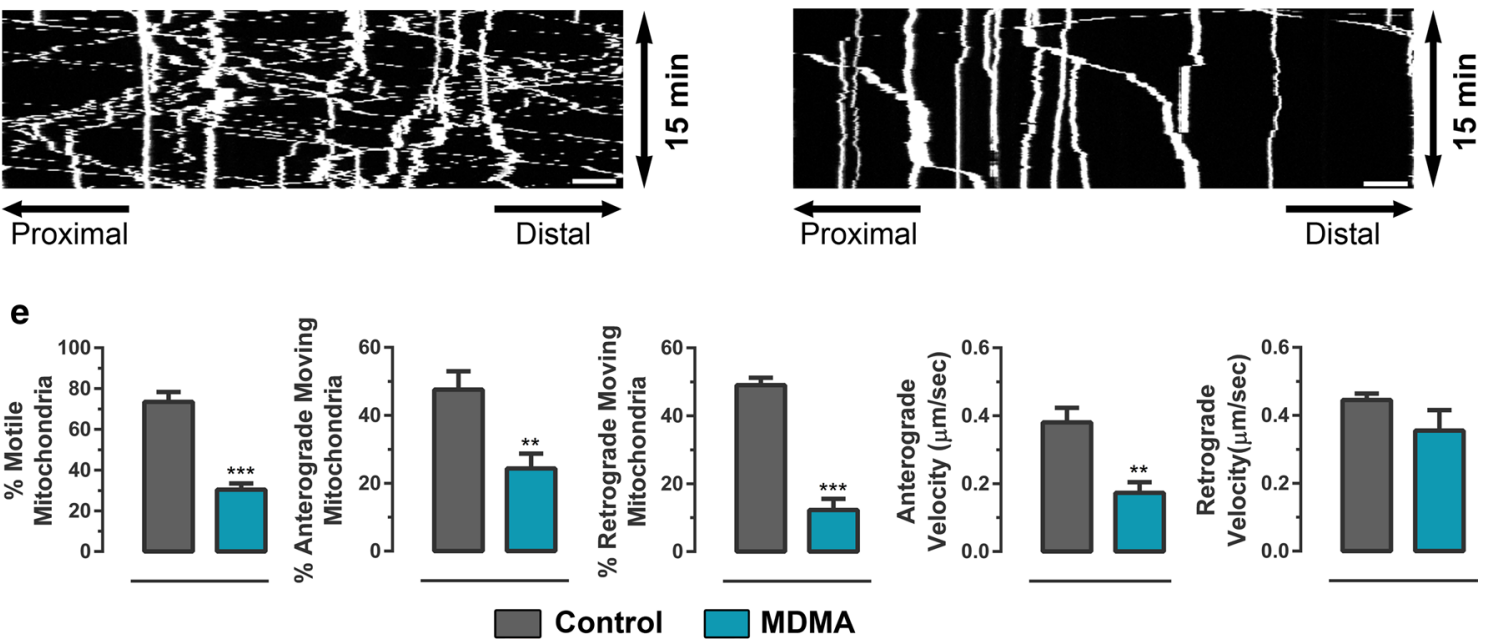

Fig. 2 Mitochondrial neuronal trafficking impairment associated with MDMA. Series of four representative confocal images, taken every $30 \mathrm{~s}$, of a live axon over-expressing MitDsRed under control conditions (a) or after $90 \mathrm{~min}$ of exposure to MDMA (1.6 mM) (b). Colored arrows indicate the same mitochondrion through the different acquisitions. Representative kymographs of a single axon under control conditions (c) or after exposure to MDMA (d). e Graphical representation of the percentage of motile mitochondria and velocities, measured in kymographs obtained before and after exposure to MDMA $(1.6 \mathrm{mM})$ for $90 \mathrm{~min}$. Data represent mean \pm SEM $(* * p<0.01 ; * * * p<0.001$ MDMA versus control, Mann-Whitney test). Scale bar $10 \mu \mathrm{m}$ (color figure online) 
the anterograde velocity, was observed (Fig. 2 and Supplementary Movie 2).

Mitochondrial dynamics in neurons is exquisitely controlled by a complex machinery involving the Miro/Trak2 complex (Macaskill et al. 2009; Sheng and Cai 2012; Wang and Schwarz 2009) and the GTPase proteins Mitofusins and Drp1 (Misko et al. 2010, 2012; Sheng and Cai 2012), in a way that depends on $\mathrm{Ca}^{2+}$ concentration (Cereghetti et al. 2008; Macaskill et al. 2009; Wang and Schwarz 2009). Exposure to $1.6 \mathrm{mM}$ MDMA increased $\mathrm{Ca}^{2+}$ concentrations in the cell body and neuronal extensions (Fig. 3a). To address whether the $\mathrm{Ca}^{2+}$-binding domains of Miro1 mediated MDMA effects, we transfected hippocampal neurons with Miro1 and also with a Miro1 construct lacking the $\mathrm{EF} \mathrm{Ca}^{2+}$-binding domains (Miro1 $\triangle \mathrm{EF}$ ), which acts as a dominant negative (Macaskill et al. 2009). However, neither the over-expression of Miro1 nor Miro1 $\Delta \mathrm{EF}$ reversed the effects of MDMA on mitochondrial neuronal trafficking. This finding, therefore, indicates a Miro1-independent mechanism for the reported trafficking impairment induced by MDMA (Fig. 3b-d).

Exposure to neurotoxins (e.g., $\beta$-amyloid protein) causes Tau-dependent impairment of mitochondrial transport (Vossel et al. 2010). We, thus, screened whether Tau was involved in MDMA-mediated effects on mitochondrial trafficking. Western blotting analysis showed increased
Fig. 4 MDMA impairs mitochondrial neuronal trafficking by a Tauand GSK3 $\beta$-dependent mechanism. Representative Western blot of Tau phosphorylation on residue $\operatorname{Thr} 181$ (a) and respective densitometric analysis (b) after exposure to MDMA (1.6 mM) for $90 \mathrm{~min}$. Data represent mean \pm SEM of three independent experiments $(* p<0.05$ MDMA vesus control, unpaired Student's $t$ test). Representative kymographs from wild-type (c), Tau $(-/-)$ (d)- or GSK3 $\beta$ Aln9-over-expressing (e) neurons. Graphical representation of the percentage of motile mitochondria and velocities, measured in kymographs obtained from Tau (-I-) (f) or GSK3 $\beta$ Aln9-over-expressing neurons (g) after exposure to MDMA $(1.6 \mathrm{mM})$ for $90 \mathrm{~min}$. Data represent mean \pm SEM $[* p<0.05 ; * * p<0.01 ; * * * p<0.001$; $* * * * p<0.0001$ MDMA versus control; ${ }^{\#} p<0.05$ Tau $(-/-) /$ GSK3 $\beta$ Aln9-over-expressing versus control neurons, two-way ANOVA and Bonferroni's post hoc test]. Scale bar $10 \mu \mathrm{m}$

Tau phosphorylation after exposure to MDMA (1.6 mM) for $90 \mathrm{~min}$, particularly in Thr181 residue (Fig. 4a, b). This observation is consistent with the findings of previous in vivo studies, in which increased Tau phosphorylation was observed in mice's hippocampus after MDMA administration (Busceti et al. 2008). By using Tau (-/-) neurons, we found that the lack of Tau protein partially abolished the MDMA-induced deficits on mitochondrial transport, thereby indicating a Tau-dependent mechanism (Fig. 4d, f).

In neurons, Tau phosphorylation is largely dependent on glycogen synthase kinase $3 \beta$ (GSK3 $\beta$ ) activity (Busceti et al. 2008; Llorens-Martín et al. 2011; Park et al. 2012). To

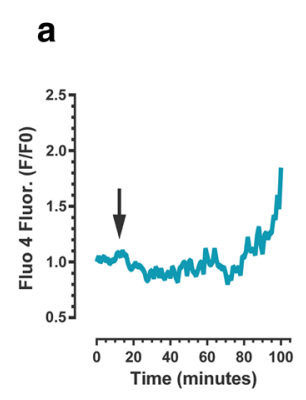

d

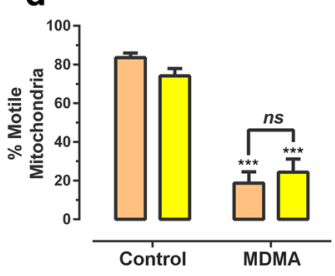

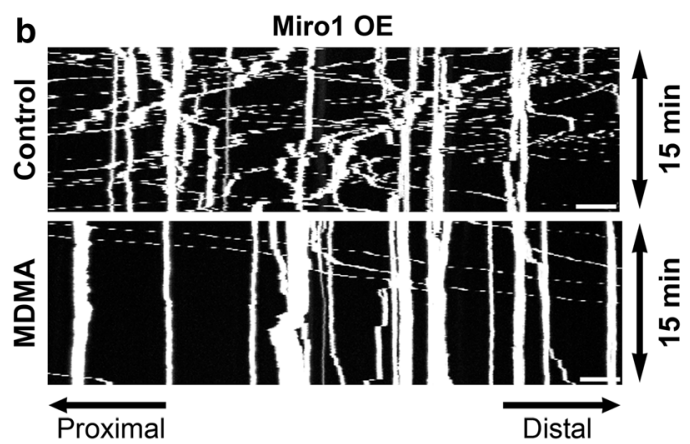

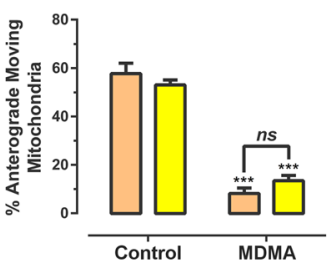

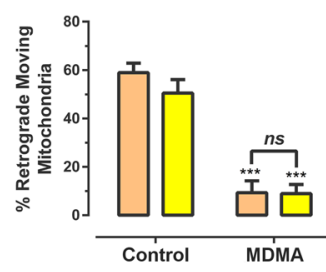

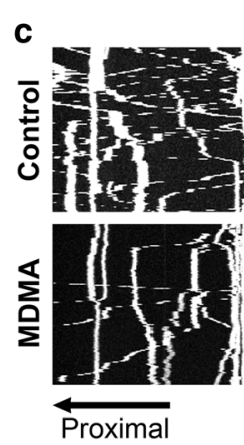

Miro1 $\triangle E F$ OE

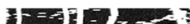
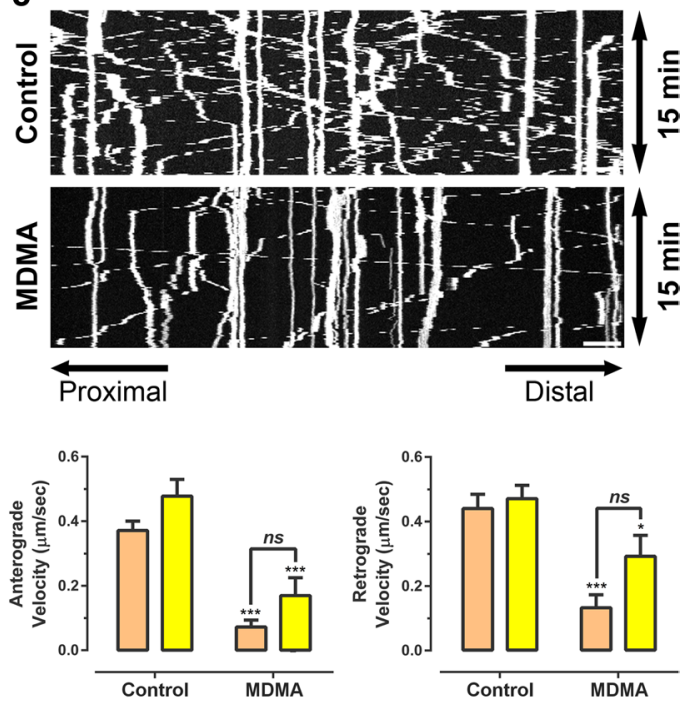

Miro1 OE

\section{Miro1 $\triangle E F$ OE}

Fig. 3 MDMA impairs mitochondrial neuronal trafficking by a $\mathrm{Ca}^{2+}$-regulated Miro1-independent mechanism. a Graphical representation of individual changes in Fluo4 whole-cell F/F0 fluorescence ratios for a single neuron, preloaded with Fluo4/AM $(5 \mu \mathrm{M})$, during $90 \mathrm{~min}$ of exposure to MDMA (1.6 mM), showing a time-dependent increase in intracellular $\mathrm{Ca}^{2+}$ concentration. The arrow indicates the onset of MDMA addition. Representative kymographs from neurons transfected with Miro1 (b) or Miro1 $\triangle \mathrm{EF}$ (c) under control conditions or after exposure to MDMA (1.6 mM) for $90 \mathrm{~min}$. d Graphical representation of the percentage of motile mitochondria and velocities, measured in kymographs obtained under control conditions or after exposure to MDMA (1.6 mM) for $90 \mathrm{~min}$. Data represent mean \pm SEM $(* p<0.05 ; * * * p<0.001$, MDMA versus control, twoway ANOVA and Bonferroni's post hoc test). Scale bar $10 \mu \mathrm{m}$ 

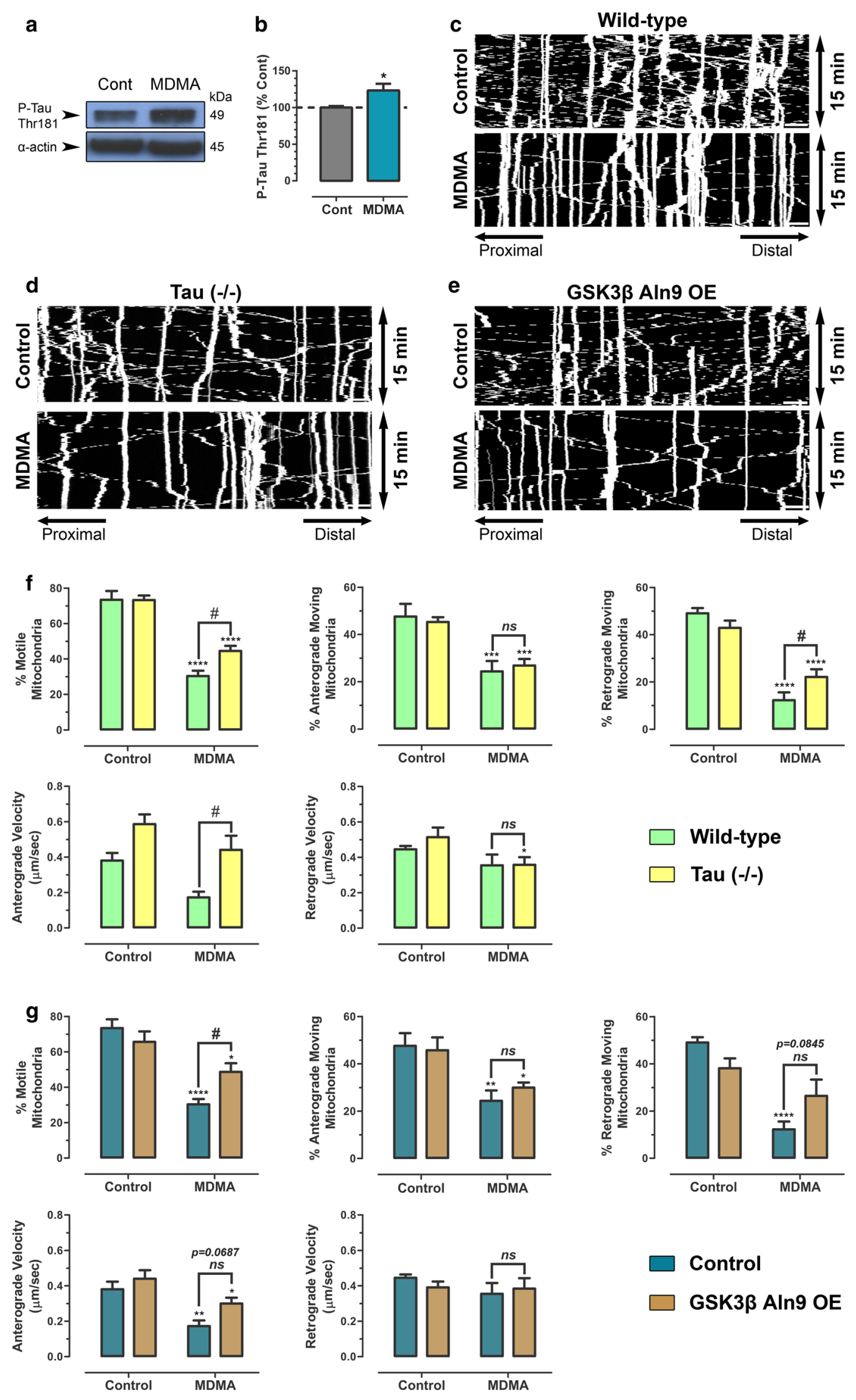

Wild-type

Tau (-I-)

Control

GSK3ß Aln9 OE 

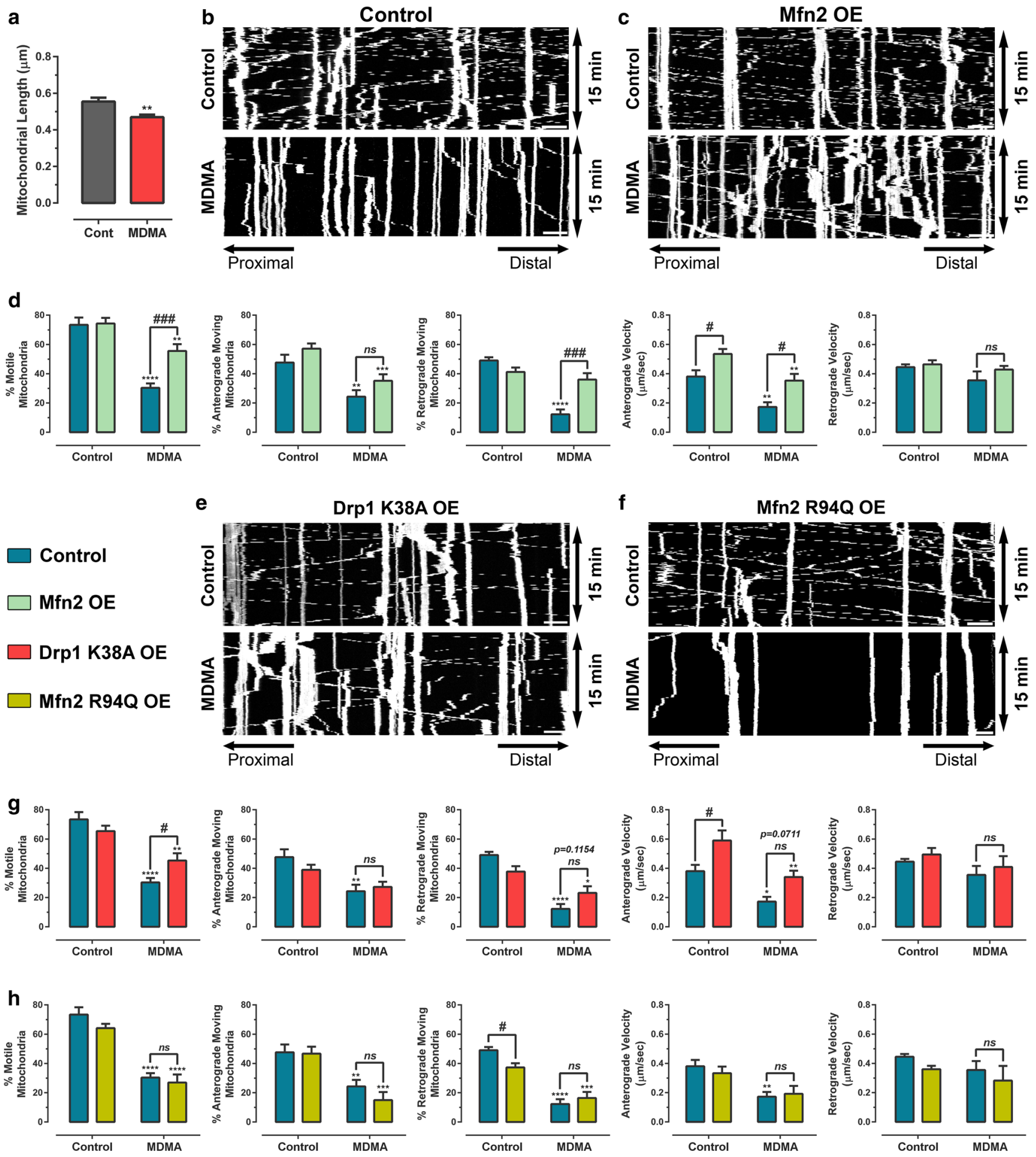

Fig. 5 MDMA impairs mitochondrial neuronal trafficking by a mitochondrial fusion/fission machinery-dependent mechanism. a Graphical representation of mitochondrial length, showing increased mitochondrial fragmentation after exposure to MDMA (1.6 mM) for $90 \mathrm{~min}$. Data represent mean \pm SEM of 126-131 individual mitochondria (29-31 axons) from three independent experiments (**p $<0.01$ MDMA versus control, Mann-Whitney test). Representative kymographs from control (b), Mitofusin2- (c), Drp1
K38A- (e) or Mitofusin2 R94Q-over-expressing neurons (f). Graphical representation of the percentage of motile mitochondria and velocities, measured in kymographs obtained from neurons overexpressing Mitofusin2 (d), Drp1 K38A (g) or Mitofusin2 R94Q (h). Data represent mean \pm SEM $[* p<0.05 ; * * p<0.01 ; * * * p<0.001$; $* * * * p<0.0001$ MDMA versus control; ${ }^{\#} p<0.05$; ${ }^{\# \# \# p} p<0.001$ Mitofusin2/Drp1 K38A-over-expressing versus control neurons, twoway ANOVA and Bonferroni's post hoc test]. Scale bar $10 \mu \mathrm{m}$ 
evaluate the involvement of GSK3 $\beta$ on the trafficking deficits elicited by MDMA, hippocampal neurons were further transfected with the dominant negative construct GSK3 $\beta$ Aln9, which lacks kinase activity (Byun et al. 2012). This experimental design resulted in a partial reversion of the MDMA-induced deficits on mitochondrial transport, comparable to those observed in Tau $(-/-$ ) neurons (Fig. 4e, g). These observations indicate, therefore, that the trafficking deficits mediated by MDMA partially depended on Tau protein and GSK3 $\beta$ activity.

During the course of the experiments, we noted that neurons exposed to MDMA exhibited smaller mitochondria, compared to control conditions (Fig. 5a), suggesting the involvement of mitochondrial fusion/fission events in the MDMA-induced mitochondrial trafficking impairment. The GTPase proteins Mitofusins and Drp1 are known to mediate these processes (Misko et al. 2010, 2012; Sheng and Cai 2012). Therefore, to evaluate the involvement of mitochondrial fission/fusion machinery on the trafficking deficits mediated by MDMA, hippocampal neurons were further transfected with Mitofusin2 or Drp1 constructs. As shown in Fig. 5c, d, Mitofusin2 overexpression almost completely rescued the mitochondrial trafficking impairment induced by MDMA. In the same way, over-expression of the dominant negative Drp1 construct (Drp1 K38A), which down-regulates mitochondrial fission (Zhao et al. 2011), also partially abolished the trafficking deficits caused by MDMA (Fig. 5e, g). Finally, over-expression of the Mitofusin2 R94Q construct, a Charcot-Marie-Tooth disease mutant with impaired fusion and transport properties (Detmer and Chan 2007; Misko et al. 2010), did not rescue the MDMA's phenotype (Fig. 5f, h). Therefore, these experiments indicate that Mitofusin2 and Drp1 largely mediate the mitochondrial trafficking deficits induced by MDMA.

\section{Discussion}

In the present study, through in situ functional experiments, we found that MDMA dramatically impaired axonal transport of mitochondria in cultured hippocampal neurons. By using Tau (-/-) hippocampal neurons, we also showed the involvement of Tau protein on the reported trafficking deficits, which were also dependent on GSK3 $\beta$ activity. Furthermore, we found that these trafficking abnormalities were strongly dependent on Mitofusin2 and Drp1 proteins, which regulate mitochondrial fission/fusion events, but not on Miro1.

As mentioned before, drugs of abuse cause a variety of complex pathophysiological events that contribute to both acute and long-term neurotoxic effects and addiction (Capela et al. 2009; Creed and Lüscher 2013; Feng and
Nestler 2013; Lüscher 2013; Lüscher and Malenka 2011). Recently, disrupted in schizophrenia-1 (DISC1), a candidate susceptibility gene for schizophrenia and major mental illness, which plays a role in brain development and synaptic function, has been shown to regulate intracellular trafficking of mitochondria in neurons (Atkin et al. 2013). Furthermore, previous studies have reported mitochondrial deficits induced by MDMA, including ATP depletion (Darvesh and Gudelsky 2005), inhibition of mitochondrial proteins (Puerta et al. 2010; Quinton and Yamamoto 2006), oxidation of mitochondrial macromolecules (Alves et al. 2007, 2009b) and, notably, deletions in mitochondrial DNA (Alves et al. 2007, 2009a), which have been mainly linked to neurotoxicity. Here, we show, for the first time, that MDMA dramatically impaired axonal transport of mitochondria in cultured hippocampal neurons. High energy demands and the requirement to maintain cellular homeostasis at sites distant to the cell body make neurons particularly vulnerable to mitochondrial transport dysfunction (Deheshi et al. 2013; Itoh et al. 2013). Therefore, considering the critical dependence of neuronal activity on mitochondrial transport, energy supply and $\mathrm{Ca}^{2+}$ buffering (MacAskill and Kittler 2010; Sheng and Cai 2012), the present results provide a new, and important, insight into the context of MDMA-induced deleterious effects to the central nervous system and suggest that alterations in mitochondrial trafficking may contribute to the acute and long-lasting neuronal effects of MDMA.

Our results show that MDMA triggered an increase in intracellular $\mathrm{Ca}^{2+}$ levels. $\mathrm{A} \mathrm{Ca}^{2+}$-dependent arrest of mitochondrial motility has been described in neurons involving the $\mathrm{EF}$ hand containing mitochondrial outer membrane protein Miro1/2 (Macaskill et al. 2009; Wang and Schwarz 2009). However, our experiments over-expressing Miro1 protein or their mutant construct Miro1 $\triangle \mathrm{EF}$, which lacks the $\mathrm{EF} \mathrm{Ca}{ }^{2+}$-binding domains (Macaskill et al. 2009), showed that the trafficking deficits caused by MDMA were not reversed. Therefore, these results indicate that MDMAinduced impaired mitochondrial trafficking most likely relied on Miro1-independent mechanisms.

Some studies have also suggested that increased expression or altered microtubule binding of Tau protein may compromise mitochondrial transport (Kopeikina et al. 2011). Furthermore, the phosphorylation levels of Tau protein appear to be critical in regulating mitochondrial neuronal trafficking (Llorens-Martín et al. 2011; Morel et al. 2010; Vossel et al. 2010). Here, we show that Tau protein was involved in the trafficking deficits elicited by MDMA, as confirmed by the partial reversion of mitochondrial deficits in Tau (-/-) neurons. Moreover, our results indicate that MDMA triggered Tau phosphorylation and that the main kinase for Tau protein, GSK3 $\beta$, is likely to be also involved, as transfection with the GSK3 $\beta$-kinase dead 
construct (GSK3 $\beta$ Aln9) reduced MDMA-induced mitochondrial trafficking alterations.

In this study, we also found that the over-expression of Mitofusin2 almost completely rescued the trafficking deficits induced by MDMA. These results suggest that a deregulation on mitochondrial fission/fusion events, involving Mitofusin2, may be an important factor contributing to the reported trafficking deficits. Although it is clear that mitochondrial fission/fusion plays a major role in quality control regulation of dysfunctional mitochondria (Itoh et al. 2013; Saxton and Hollenbeck 2012; Shutt and McBride 2013), an interplay between mitochondrial fission/fusion events and mitochondrial transport has also been described (Kageyama et al. 2012; Li et al. 2004; Misko et al. 2010, 2012; Verstreken et al. 2005). Interestingly, the loss of Mitofusin2 caused abnormal mitochondrial morphology and transport, respiratory chain dysfunction and severe lack of dopaminergic terminals in the striatum (Lee et al. 2012). The Mitofusin2 R94Q mutant is a CMT2A mutant with impaired fusion (Detmer and Chan 2007) and transport properties (Misko et al. 2010, 2012). Since this mutant protein acts as a dominant negative, the physiological activity of Mitofusin2 is repressed, though endogenous fission machinery is not affected (Detmer and Chan 2007). Thus, our over-expression experiments with the Mitofusin2 R94Q mutant, which did not rescue MDMA-mediated deficits, indicate that fully functional Mitofusin2 was required to reverse MDMA's effects and suggest that MDMA may alter fusion mechanisms. Moreover, our data with the Drp1 K38A mutant, which inhibits endogenous fission without affecting mitochondrial fusion (Zhao et al. 2011), support also a role for fission events in MDMA-regulated phenotype. Therefore, these experiments indicate that Mitofusin2 and Drp1 largely mediated the mitochondrial trafficking deficits induced by MDMA and suggest that MDMA may alter the mitochondrial fusion/fission equilibrium.

In this study, MDMA reduced the percentage of motile mitochondria in both anterograde and retrograde directions, as well as the anterograde velocity, though the retrograde mitochondrial velocity was not significantly changed, even after 120 min of incubation. A previous study demonstrated that kinesin- 1 and dynein strongly affected the frequency of mitochondria movement, but not the mitochondrial speed (van Spronsen et al. 2013). The reduction in the percentage of mitochondrial motility in both directions is compatible with MDMA effects on Tau protein, with consequent destabilization of microtubules, and in the mitochondrial fusion/fission equilibrium, which may modify the interaction between microtubules and motor proteins, thus disrupting mitochondrial translocation. Another study, analyzing the influence of mitochondrial membrane potential on transport, showed that mitochondria with low potential are selectively transported toward the cell body (Miller and Sheetz 2004). Since the loss of serotonergic nerve terminals in MDMA-administrated rats is by far the most studied neurotoxic event (Capela et al. 2009), it is expected that MDMA may damage terminal mitochondria, which, in turn, move toward the cell body for regeneration or degradation. Additionally, it was demonstrated that the knockdown of myosin VI led to an increase in the retrograde mitochondrial velocity, whereas no effect was seen on anterograde direction (Pathak et al. 2010). Therefore, these mechanisms may justify the differential effect of MDMA on mitochondrial transport velocity.

To the best of our knowledge, this study provides the first indication that a drug of abuse dramatically alters mitochondrial neuronal trafficking and dynamics. As mitochondrial transport and targeting to neuronal compartments and synapses is essential for many neuronal functions, including energy production and correct synaptic transmission (López-Doménech et al. 2012; Sheng and Cai 2012; Wang and Schwarz 2009), our findings underscore a novel mechanism of action of MDMA. We postulate that this mechanism may contribute to the MDMA neuronal effects, including its psychostimulant, addictive and neurotoxic actions.

Acknowledgments We thank Luca Scorrano (University of Geneva) and Miquel Vila (VHIR, Barcelona) for providing the Mitofusin and Drp1 constructs, and Isidre Ferrer (University of Barcelona) for providing the P-Tau Thr181 antibody. This study was supported by grant BFU2008-3980 ("Ministerio de Ciencia e Innovacion" (MICINN), Spain) and a grant from the "Plan Nacional de Drogas" to ES, and by the "Fundação para a Ciência e Tecnologia (FCT)," Portugal (Project PTDC/SAU-FCF/102958/2008), under the framework of the "Programa Operacional Temático Factores de Competitividade (COMPTE) do Quadro Comunitário de Apoio III" and "Fundo Comunitário Europeu (FEDER)" (FCOMP-01-0124-FEDER-011079). DJB's stay at IRB Barcelona was partially supported by FCT, Portugal. DJB was supported by fellowship (SFRH/BD/64939/2009) from FCT, Portugal.

Conflict of interest The authors declare that they have no conflict of interest.

\section{References}

Alves E, Summavielle T, Alves CJ et al (2007) Monoamine oxidase$\mathrm{B}$ mediates ecstasy-induced neurotoxic effects to adolescent rat brain mitochondria. J Neurosci 27:10203-10210

Alves E, Binienda Z, Carvalho F et al (2009a) Acetyl-1-carnitine provides effective in vivo neuroprotection over 3,4-methylenedioximethamphetamine-induced mitochondrial neurotoxicity in the adolescent rat brain. Neuroscience 158:514-523

Alves E, Summavielle T, Alves CJ et al (2009b) Ecstasy-induced oxidative stress to adolescent rat brain mitochondria in vivo: influence of monoamine oxidase type A. Addict Biol 14:185-193

Atkin TA, MacAskill AF, Brandon NJ, Kittler JT (2013) Disrupted in schizophrenia-1 regulates intracellular trafficking of mitochondria in neurons. Mol Psychiatry 16:122-124 
Bai F, Jones DC, Lau SS, Monks TJ (2001) Serotonergic neurotoxicity of 3,4-( \pm -methylenedioxyamphetamine and 3,4-( \pm -methylendioxymethamphetamine (ecstasy) is potentiated by inhibition of $\gamma$-glutamyl transpeptidase. Chem Res Toxicol 14:863-870

Barbosa DJ, Capela JP, Oliveira JMA et al (2012) Pro-oxidant effects of Ecstasy and its metabolites in mouse brain synaptosomes. Br J Pharmacol 165:1017-1033

Barbosa DJ, Capela JP, Silva R et al (2014a) "Ecstasy"-induced toxicity in SH-SY5Y differentiated cells: role of hyperthermia and metabolites. Arch Toxicol 88:515-531

Barbosa DJ, Capela JP, Silva R et al (2014b) The mixture of "ecstasy" and its metabolites is toxic to human SH-SY5Y differentiated cells at in vivo relevant concentrations. Arch Toxicol 88:455-473

Busceti CL, Biagioni F, Riozzi B et al (2008) Enhanced tau phosphorylation in the hippocampus of mice treated with 3,4-methylenedioxymethamphetamine ("ecstasy"). J Neurosci 28:3234-3245

Byun J, Kim BT, Kim YT, Jiao Z, Hur E, Zhou F (2012) Slit2 inactivates GSK3 $\beta$ to signal neurite outgrowth inhibition. PLoS One 7:e51895

Capela JP, Ruscher K, Lautenschlager M et al (2006) Ecstasy-induced cell death in cortical neuronal cultures is serotonin 2A-receptordependent and potentiated under hyperthermia. Neuroscience 139:1069-1081

Capela JP, Macedo C, Branco PS et al (2007) Neurotoxicity mechanisms of thioether ecstasy metabolites. Neuroscience 146:1743-1757

Capela JP, Carmo H, Remião F, Bastos ML, Meisel A, Carvalho F (2009) Molecular and cellular mechanisms of ecstasy-induced neurotoxicity: an overview. Mol Neurobiol 39:210-271

Capela JP, da Costa Araújo S, Costa VM et al (2013) The neurotoxicity of hallucinogenic amphetamines in primary cultures of hippocampal neurons. Neurotoxicology 34:254-263

Carvalho M, Remiao F, Milhazes N et al (2004) The toxicity of $\mathrm{N}$-methyl-alpha-methyldopamine to freshly isolated rat hepatocytes is prevented by ascorbic acid and $\mathrm{N}$-acetylcysteine. Toxicology 200:193-203

Cereghetti GM, Stangherlin A, de Brito OM et al (2008) Dephosphorylation by calcineurin regulates translocation of Drp1 to mitochondria. Proc Natl Acad Sci USA 105:15803-15808

Creed MC, Lüscher C (2013) Drug-evoked synaptic plasticity: beyond metaplasticity. Curr Opin Neurobiol 23:553-558

Darvesh AS, Gudelsky GA (2005) Evidence for a role of energy dysregulation in the MDMA-induced depletion of brain 5-HT. Brain Res 1056:168-175

de Barreda EG, Pérez M, Ramos PG et al (2010) Tau-knockout mice show reduced GSK3-induced hippocampal degeneration and learning deficits. Neurobiol Dis 37:622-629

Deheshi S, Pasqualotto BA, Rintoul GL (2013) Mitochondrial trafficking in neuropsychiatric diseases. Neurobiol Dis 51:66-71

Detmer SA, Chan DC (2007) Complementation between mouse Mfn1 and Mfn2 protects mitochondrial fusion defects caused by CMT2A disease mutations. J Cell Biol 176:405-414

Feng J, Nestler EJ (2013) Epigenetic mechanisms of drug addiction. Curr Opin Neurobiol 23:521-528

Granado N, Ares-Santos S, Oliva I et al (2011) Dopamine D2-receptor knockout mice are protected against dopaminergic neurotoxicity induced by methamphetamine or MDMA. Neurobiol Dis 42:391-403

Itoh K, Nakamura K, Iijima M, Sesaki H (2013) Mitochondrial dynamics in neurodegeneration. Trends Cell Biol 23:64-71

Kageyama Y, Zhang Z, Roda R et al (2012) Mitochondrial division ensures the survival of postmitotic neurons by suppressing oxidative damage. J Cell Biol 197:535-551

Kenny PJ (2011) Common cellular and molecular mechanisms in obesity and drug addiction. Nat Rev Neurosci 12:638-651

Kopeikina KJ, Carlson GA, Pitstick R et al (2011) Tau accumulation causes mitochondrial distribution deficits in neurons in a mouse model of tauopathy and in human Alzheimer's disease grain. Am J Pathol 179:2071-2082

Lee S, Sterky FH, Mourier A et al (2012) Mitofusin 2 is necessary for striatal axonal projections of midbrain dopamine neurons. Hum Mol Genet 21:4827-4835

Li Z, Okamoto K, Hayashi Y, Sheng M (2004) The importance of dendritic mitochondria in the morphogenesis and plasticity of spines and synapses. Cell 119:873-887

Llorens-Martín M, López-Doménech G, Soriano E, Avila J (2011) GSK3 $\beta$ is involved in the relief of mitochondria pausing in a taudependent manner. PLoS One 6:e27686

López-Doménech G, Serrat R, Mirra S et al (2012) The Eutherian Armcx genes regulate mitochondrial trafficking in neurons and interact with Miro and Trak2. Nat Commun 3:814

Lüscher C (2013) Cocaine-evoked synaptic plasticity of excitatory transmission in the ventral tegmental area. Cold Spring Harb Perspect Med 3:a012013

Lüscher C, Malenka RC (2011) Drug-evoked synaptic plasticity in addiction: from molecular changes to circuit remodeling. Neuron 69:650-663

MacAskill AF, Kittler JT (2010) Control of mitochondrial transport and localization in neurons. Trends Cell Biol 20:102-112

Macaskill AF, Rinholm JE, Twelvetrees AE et al (2009) Miro1 is a calcium sensor for glutamate receptor-dependent localization of mitochondria at synapses. Neuron 61:541-555

MacAskill AF, Atkin TA, Kittler JT (2010) Mitochondrial trafficking and the provision of energy and calcium buffering at excitatory synapses. Eur J Neurosci 32:231-240

Miller KE, Sheetz MP (2004) Axonal mitochondrial transport and potential are correlated. J Cell Sci 117:2791-2804

Misko A, Jiang S, Wegorzewska I, Milbrandt J, Baloh RH (2010) Mitofusin 2 is necessary for transport of axonal mitochondria and interacts with the Miro/Milton complex. J Neurosci 30:4232-4240

Misko AL, Sasaki Y, Tuck E, Milbrandt J, Baloh RH (2012) Mitofu$\sin 2$ mutations disrupt axonal mitochondrial positioning and promote axon degeneration. J Neurosci 32:4145-4155

Morel M, Authelet M, Dedecker R, Brion JP (2010) Glycogen synthase kinase- $3 \beta$ and the $\mathrm{p} 25$ activator of cyclin dependent kinase 5 increase pausing of mitochondria in neurons. Neuroscience 167:1044-1056

Park H, Kam T, Kim Y et al (2012) Neuropathogenic role of adenylate kinase-1 in $A \beta$-mediated tau phosphorylation via AMPK and GSK3 $\beta$. Hum Mol Genet 21:2725-2737

Pathak D, Sepp KJ, Hollenbeck PJ (2010) Evidence that myosin activity opposes microtubule-based axonal transport of mitochondria. J Neurosci 30:8984-8992

Perier C, Vila M (2012) Mitochondrial biology and Parkinson's disease. Cold Spring Harb Perspect Med 2:a009332

Puerta E, Hervias I, Goñi-Allo B et al (2010) Methylenedioxymethamphetamine inhibits mitochondrial complex I activity in mice: a possible mechanism underlying neurotoxicity. Br J Pharmacol 160:233-245

Quinton MS, Yamamoto BK (2006) Causes and consequences of methamphetamine and MDMA toxicity. AAPS J 8:337-347

Ricaurte GA, Yuan J, McCann UD (2000) ( \pm )3,4-Methylenedioxymethamphetamine ('ecstasy')-induced serotonin neurotoxicity: studies in animals. Neuropsychobiology 42:5-10

Rintoul GL, Reynolds IJ (2010) Mitochondrial trafficking and morphology in neuronal injury. Biochim Biophys Acta 1802:143-150

Saxton WM, Hollenbeck PJ (2012) The axonal transport of mitochondria. J Cell Sci 125:2095-2104

Shahpasand K, Uemura I, Saito T et al (2012) Regulation of mitochondrial transport and inter-microtubule spacing by tau phosphorylation at the sites hyperphosphorylated in Alzheimer's disease. J Neurosci 32:2430-2441 
Shao L, Martin MV, Watson SJ et al (2008) Mitochondrial involvement in psychiatric disorders. Ann Med 40:281-295

Sheng Z, Cai Q (2012) Mitochondrial transport in neurons: impact on synaptic homeostasis and neurodegeneration. Nat Rev Neurosci 13:77-93

Shutt TE, McBride HM (2013) Staying cool in difficult times: mitochondrial dynamics, quality control and the stress response. Biochim Biophys Acta 1833:417-424

Simantov R, Tauber M (1997) The abused drug MDMA (ecstasy) induces programmed death of human serotonergic cells. FASEB J 11:141-146

van Spronsen M, Mikhaylova M, Lipka J et al (2013) TRAK/Milton motor-adaptor proteins steer mitochondrial trafficking to axons and dendrites. Neuron 77:485-502

Verstreken P, Ly CV, Venken KJT, Koh T, Zhou Y, Bellen HJ (2005) Synaptic mitochondria are critical for mobilization of reserve pool vesicles at drosophila neuromuscular junctions. Neuron 47:365-378

Vossel KA, Zhang K, Brodbeck J et al (2010) Tau reduction prevents A $\beta$-induced defects in axonal transport. Science 330:198

Wang X, Schwarz TL (2009) The mechanism of $\mathrm{Ca}^{2+}$-dependent regulation of kinesin-mediated mitochondrial motility. Cell 136:163-174

Wang DB, Garden GA, Kinoshita C et al (2013) Declines in Drp1 and Parkin expression underlie DNA damage-induced changes in mitochondrial length and neuronal death. J Neurosci 33:1357-1365

Zhao J, Liu T, Jin S et al (2011) Human MIEF1 recruits Drp1 to mitochondrial outer membranes and promotes mitochondrial fusion rather than fission. EMBO J 30:2762-2778 